\title{
Investigating the complex genetic architecture of ankle-brachial index, a measure of peripheral arterial disease, in non-Hispanic whites
}

\author{
Sharon LR Kardia*†1, M Todd Greene ${ }^{\dagger 1}$, Eric Boerwinkle ${ }^{2}$, Stephen T Turner ${ }^{3}$ \\ and Iftikhar J Kullo 4
}

Address: ${ }^{1}$ Department of Epidemiology, University of Michigan, Ann Arbor, Michigan 48109, USA, ${ }^{2}$ Human Genetics Center and Institute of Molecular Medicine, University of Texas-Houston Health Science Center, Houston, Texas 77030, USA, ${ }^{3}$ Division of Nephrology and Hypertension, and the Department of Internal Medicine, Mayo Clinic, Rochester, Minnesota 55905, USA and ${ }^{4}$ Division of Cardiovascular Diseases, Department of Internal Medicine, Mayo Clinic, Rochester, Minnesota 55905, USA

Email: Sharon LR Kardia* - skardia@umich.edu; M Todd Greene - mtgreene@umich.edu; Eric Boerwinkle - Eric.Boerwinkle@uth.tmc.edu; Stephen T Turner - sturner@mayo.edu; Iftikhar J Kullo - kullo.iftikhar@mayo.edu

* Corresponding author †Equal contributors

Published: I5 May 2008

BMC Medical Genomics 2008, I:16 doi:10.1 186/1755-8794-1-16

This article is available from: http://www.biomedcentral.com/I755-8794/I/16

(c) 2008 Kardia et al; licensee BioMed Central Ltd.

This is an Open Access article distributed under the terms of the Creative Commons Attribution License (http://creativecommons.org/licenses/by/2.0), which permits unrestricted use, distribution, and reproduction in any medium, provided the original work is properly cited.

\begin{abstract}
Background: Atherosclerotic peripheral arterial disease (PAD) affects 8-10 million people in the United States and is associated with a marked impairment in quality of life and an increased risk of cardiovascular events. Noninvasive assessment of PAD is performed by measuring the ankle-brachial index $(A B I)$. Complex traits, such as $A B I$, are influenced by a large array of genetic and environmental factors and their interactions. We attempted to characterize the genetic architecture of $A B I$ by examining the main and interactive effects of individual single nucleotide polymorphisms (SNPs) and conventional risk factors.
\end{abstract}

Methods: We applied linear regression analysis to investigate the association of 435 SNPs in I I 2 positional and biological candidate genes with $\mathrm{ABI}$ and related physiological and biochemical traits in 1046 non-Hispanic white, hypertensive participants from the Genetic Epidemiology Network of Arteriopathy (GENOA) study. The main effects of each SNP, as well as SNP-covariate and SNP-SNP interactions, were assessed to investigate how they contribute to the inter-individual variation in ABI. Multivariable linear regression models were then used to assess the joint contributions of the top SNP associations and interactions to $A B I$ after adjustment for covariates. We reduced the chance of false positives by I) correcting for multiple testing using the false discovery rate, 2) internal replication, and 3) four-fold cross-validation.

Results: When the results from these three procedures were combined, only two SNP main effects in NOS3, three SNPcovariate interactions (ADRB2 Gly 16 - lipoprotein(a) and SLC4A5 - diabetes interactions), and 25 SNP-SNP interactions (involving SNPs from 29 different genes) were significant, replicated, and cross-validated. Combining the top SNPs, risk factors, and their interactions into a model explained nearly $18 \%$ of variation in $A B I$ in the sample. SNPs in six genes (ADD2, ATP6VIBI, PRKAR2B, SLCI7A2, SLC22A3, and TGFB3) were also influencing triglycerides, C-reactive protein, homocysteine, and lipoprotein(a) levels.

Conclusion: We found that candidate gene SNP main effects, SNP-covariate and SNP-SNP interactions contribute to the inter-individual variation in $\mathrm{ABI}$, a marker of PAD. Our findings underscore the importance of conducting systematic investigations that consider context-dependent frameworks for developing a deeper understanding of the multidimensional genetic and environmental factors that contribute to complex diseases. 


\section{Background}

Atherosclerotic peripheral arterial disease (PAD) affects 8-10 million people in the United States $[1,2]$ and is associated with a marked impairment in quality of life and an increased risk of stroke, myocardial infarction, and cardiovascular death [3]. Noninvasive assessment of PAD is performed by measuring the ankle-brachial index (ABI), the ratio of systolic blood pressure (SBP) at the ankle to the SBP in the arm. Normally ABI is $\geq 1.0$, but with increasing narrowing of the lumen of arteries in the lower extremities, SBP at the ankle falls. Because individuals with PAD may not have typical symptoms of exertional leg discomfort, ABI values $\leq 0.95$ or $\leq 0.90$ have been used to diagnose the presence of PAD.

Coronary artery disease, cerebrovascular disease, and PAD are manifestations of the atherosclerotic disease process. As such, many of the well-established risk factors for atherosclerosis, such as increasing age, hyperlipidemia, hypertension, cigarette smoking and diabetes [4], contribute to these diseases. While these conventional risk factors have been associated with PAD, they explain $<20 \%$ of inter-individual variation in ABI [5]. The contribution of other 'novel' biochemical and genetic risk factors is less well characterized. In particular, little is known regarding genetic factors influencing inter-individual variation in ABI.

A recent review of the few association studies conducted to date suggests that the investigations of a small number of genes have failed to uncover compelling genetic determinants of PAD and most studies have only focused on the main effects of one polymorphism per gene [6]. We have previously reported findings from an association study that examined the relationships between variations in the NOS3 gene and ABI [7]. While this investigation also focused on a single gene, it extended the literature by employing a tag SNP approach to adequately cover variation and investigated the potential influence of 14 polymorphisms and related haplotypes on inter-individual ABI variation. Our findings provided evidence that NOS3 variants may have moderate effects on $\mathrm{ABI}$ variation, which is in line with the conventional wisdom that the effect of a single gene on a complex disease is expected to be modest and that genetic susceptibility to complex atherosclerotic disease is likely polygenic [6]. Furthermore, while the single candidate gene approach, largely employed to date, may offer valuable insights into the etiology of PAD, it fails to consider the interactive and context-dependent nature that defines complex diseases like PAD.

As a part of the Genetic Epidemiology Network of Arteriopathy (GENOA) study, genetic variants in a large collection of positional and biological candidate genes have been measured to better understand the contribution of genes to risk of arteriopathies that are associated with diseases of the heart, brain, kidneys, and peripheral arteries. Even with an increased understanding of the molecular genetic and biochemical basis of blood pressure (BP) regulation, lipoprotein metabolism, inflammation, oxidative stress, and glucose metabolism, it has been difficult to predict individual susceptibility to these diseases [8]. Complex traits, such as ABI, are influenced by a large array of genetic, environmental, behavioral, and social factors and their interactions [9]. As such, in order to develop a more complete picture of genetic susceptibility to PAD, it is necessary to move beyond the exclusive investigation of single gene effects. In this paper, we begin to characterize the complex genetic architecture of $\mathrm{ABI}$ by examining the effect of individual SNPs in candidate genes, interactions between SNPs and conventional risk factors, as well as interactions between SNPs within and across genes (intragenic and intergenic epistasis). In addition, we investigated whether the SNPs affecting ABI also influence 15 physiological and biochemical correlates of the pathways underlying variation in $\mathrm{ABI}$. These include age, body mass index (BMI), smoking, SBP and diastolic blood pressure (DBP), fasting plasma cholesterol, high-density lipoprotein (HDL) cholesterol, triglycerides, C-reactive protein (CRP), homocysteine, lipoprotein (a) (Lp(a)), fibrinogen, hypertension, and diabetes. This paradigm shift to a more encompassing attempt to unravel the complex genetic architecture is an advance over the simplified single gene approach employed in the past. While difficult to dissect and interpret, a deeper understanding of interactive effects and underlying correlation structures will likely offer additional insights into the etiology of PAD and possible explanations for PAD susceptibility for certain individuals within particular contexts.

For this study, we identified 435 SNPs in 112 genes that have been previously implicated as playing a role in $\mathrm{BP}$ regulation, lipoprotein metabolism, inflammation, oxidative stress, and diabetes. To our knowledge, no other study has comprehensively investigated how this amount of variation in numerous candidate genes may influence PAD risk. A summary of the genes and their corresponding SNPs is provided [see Additional file 1]. Although association studies are favored over linkage studies for unraveling the genetic bases of complex disorders, lack of replication in a majority of such studies has been a major concern [10]. To reduce false positives we combined three approaches: adjustment for multiple testing using the false discovery rate (FDR) [11], internal replication, and cross-validation [12]. 


\section{Methods \\ Study Population}

Subjects included non-Hispanic white participants in the Genetic Epidemiology Network of Arteriopathy (GENOA) study, a community-based study of hypertensive sibships that aims to identify genes influencing BP $[13,14]$. The study was approved by the Institutional Review Board of Mayo Clinic, Rochester MN. Written informed consent was obtained from each participant. In the initial phase of the GENOA study (9/1995 to 6/2001), sibships containing $\geq 2$ individuals with essential hypertension diagnosed before age 60 years were selected for participation. At the Rochester, MN field center, 1583 non-Hispanic whites were enrolled. Participants returned in Phase II of GENOA for physical examination, and measurement of non-conventional and novel risk factors as well as the ABI. Through November of 2004, ABI had been measured in 1046 participants.

\section{Clinical Assessments and Covariate Definitions}

Height was measured by stadiometer, weight by electronic balance, and BMI was calculated as weight in kilograms divided by the square of height in meters. Resting SBP and DBP were measured by a random zero sphygmomanometer. Blood was drawn by venipuncture after an overnight fast. Serum total cholesterol and HDL cholesterol were measured by standard enzymatic methods. Low-density lipoprotein (LDL) cholesterol levels were calculated using the Friedewald formula [15]. The diagnosis of hypertension was established based on BP levels measured at the study visit ( $\geq 140 / 90 \mathrm{mmHg}$ ) or a prior diagnosis of hypertension and current treatment with antihypertensive medications. Diabetes was considered present if the subject was being treated with insulin or oral agents or had a fasting glucose level $\geq 126 \mathrm{mg} / \mathrm{dL}$. Participants were considered as having "ever smoked" if they had smoked more than 100 cigarettes during their lifetime. CRP was measured by a highly sensitive immunoturbidimetric assay [16]. Fibrinogen was measured by the Clauss (clotting time based) method [17]. Lp(a) in serum was measured by an immunoturbidimetric assay using the $\mathrm{SPQ}^{\mathrm{TM}}$ Test System (Diasorin, Stillwater MN) as previously described [18]. Plasma homocysteine was measured by high-pressure liquid chromatography. Inter-assay coefficients of variance were: CRP, 2.6-2.8\%; fibrinogen, 5.8-6.8\%; Lp(a), 8.6-13.5\%; homocysteine, 5.7-7.4\%.

\section{Ankle-brachial index}

ABI was measured in the supine position following a 5min rest. Appropriately sized BP cuffs were placed on each arm and ankle, and a Doppler ultrasonic instrument (Medisonics, Minneapolis MN) was used to detect each pulse. The cuff was inflated to $10 \mathrm{~mm} \mathrm{Hg}$ above SBP and deflated at $2 \mathrm{~mm} \mathrm{Hg} / \mathrm{s}$. The first reappearance of the pulse was taken as the SBP. To calculate ABI, the SBP at each ankle site (posterior tibial and dorsalis pedis) was divided by the higher of the 2 brachial pressures. The lowest of the 4 ratios was designated as the ABI. The correlation of the lowest $\mathrm{ABI}$ with the average of the $2 \mathrm{ABI}$ from the same leg was 0.98, and inferences were similar using the lowest $\mathrm{ABI}$ or the average ABI.

\section{SNP Selection}

Four hundred and thirty five SNPs from 112 genes known or hypothesized to be involved in BP regulation, lipoprotein metabolism, inflammation, oxidative stress, vascular wall biology, obesity and diabetes were identified from the genetic association literature and positional candidate gene studies [19]. These biological pathways and disease conditions are related to atherosclerosis. As PAD is an atherosclerotic process, studying variations in these candidate genes may yield insights into the genetic architecture of ABI. SNPs were chosen based on a number of different criteria including the published literature, non-synonymous SNPs with a minor allele frequency (MAF) $>0.02$, and tag SNPs using public databases such as dbSNP [20] and Seattle SNPs [21].

Our algorithm for SNP selection first identified non-synonymous SNPs with a minor allele frequency (MAF) > 0.02 based on data from the Seattle SNPs database [21]. Second, we identified all SNPs with a MAF $>0.1$ and unique sequence context that could potentially be typed in any of the three ethnic groups (non-Hispanic white, African-American, Hispanic) sampled in the GENOA study [13]. From the latter SNPs, tag SNPs were selected based on the $r^{2}$ method described by Carlson et al. [22]. The final list of SNPs to be genotyped was established by selecting 1 SNP from each bin pair according to the following selection prioritization: (first) a tag SNP in a conserved region (compared to mouse); (second) a tag SNP not in a conserved region; (third) a non-tag SNP in a conserved region; (fourth) neither a tag SNP nor a SNP in a conserved region. We used this priority system because several bins had multiple tag SNPs, and some bins had no identified tag SNPs.

\section{Genotyping}

DNA was isolated using the PureGene DNA Isolation Kit from Gentra Systems (Minneapolis MN). Genotyping, based on polymerase chain reaction (PCR) amplification techniques, was conducted at the University of TexasHealth Sciences Center at Houston using the TaqMan assay and ABI Prism ${ }^{\circledR}$ Sequence Detection System (Applied Biosystems, Foster City CA). Primers and probes are available from the authors upon request. Quality control measures for genotyping assays included robotic liquid handling; separate pre- and post-PCR areas; standard protocols and quality control analyses including 5\% dupli- 
cates, positive and negative controls, computerized sample tracking, and data validity checks.

\section{Statistical Analysis}

All analyses were carried out using the R statistical language [23]. Variables with skewed distributions were log transformed. Risk factor correlations were estimated using Pearson's product moment correlation. Allele and genotype frequencies were calculated using standard gene counting methods. Linkage disequilibrium (LD), as measured by $\mathrm{r}^{2}[24]$, was estimated using an expectation maximization (EM) algorithm. Hardy-Weinberg Equilibrium was assessed using a chi-square test or Fisher's exact test if a genotype class had less than 5 individuals [25]. In all models, ABI was adjusted for age, sex, BMI, smoking status (ever vs. never), diabetes, and hypertension. Adjustment variables were chosen because they have known associations with PAD $[2,26-29]$ or because they were statistically significant predictors of $\mathrm{ABI}$ in this dataset.

In the first stage of analysis, we tested for associations of each of the predictors (SNPs and demographic/biochemical risk factors) with $\mathrm{ABI}$ using least-squares linear regression methods [30]. We also tested for association between each single SNP and each risk factor to identify potential confounders. To determine whether interactions among predictors explained additional variation in $\mathrm{ABI}$, we tested pairwise interactions among all possible pairs of predictors (i.e. SNP-SNP, SNP-risk factor, and risk factor-risk factor interactions). Associations involving interactions were assessed with a partial $\mathrm{F}$ test, which compares a full model that includes both the interaction terms and the main effects of the variables comprising the interaction terms to a reduced model that includes only the main effects.

To reduce false positives we used three different approaches: adjustment for multiple testing using FDR < 0.30 [11], internal replication with two subsets of unrelated individuals followed by testing for homogeneity of genotype-phenotype effects, and, finally, four-fold crossvalidation (repeated 10 times) [31]. To create replication subsets, we randomly selected 1 hypertensive sib from each sibship without replacement to create Subset 1 and then randomly selected another hypertensive sib from each sibship to create Subset 2. The GENOA cohort contained a small number of singletons (i.e.- no matching sibs) that were equally divided between the two samples. A dichotomous "sample" variable was generated, with all subjects in Subset 1 assigned a value of 0 and all subjects in Subset 2 assigned a value of 1. If an effect was found to be significant in both subsets, modeling an interaction term between the significant SNP and the "sample" variable was used to assess the homogeneity of the respective genotype-phenotype effect. This interaction model was then compared to a reduced model without the "sample" interaction and significance was assessed with a partial Ftest.

Cross-validation significantly reduces false positive results by eliminating associations that lack predictive ability in independent test samples. We performed four-fold crossvalidation by dividing the full sample into four equally sized groups. Three of the four groups were combined into a training dataset, and the modeling strategy outlined above was carried out to estimate model coefficients. These coefficients were then applied to the fourth group, the testing dataset, to predict the value of the outcome variable of each individual in the independent test sample. This process was repeated for each of the 4 testing sets. Predicted values for all individuals in the test set were then subtracted from their observed values, yielding the total residual variability (SSE), $\sum_{i=1}^{n}\left(y_{i}-\hat{y}_{i}\right)^{2}$. The total variability in the outcome (SST) - the difference between each individual's observed value and the mean value for the outcome - was then calculated, $\sum_{i=1}^{n}\left(\bar{y}-y_{i}\right)^{2}$. In order to estimate the proportion of variation in the outcome predicted in the independent test samples, the cross-vali-

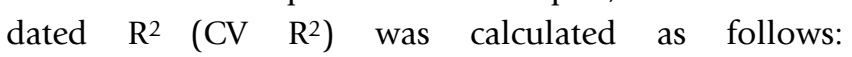
$C V R^{2}=\frac{S S T-S S E}{S S T}$. This cross-validation method provides a more accurate measure of the predictive ability of the genetic models and will be negative when the model's predictive ability is poor. Because random variations in the sampling of the four mutually exclusive test groups can potentially impact the estimates of $\mathrm{CV} \mathrm{R}^{2}$, this procedure was repeated 10 times and the $C V \mathrm{R}^{2}$ values were averaged [31]. Univariate associations were considered cross-validated if the average percent variation predicted in independent test samples was greater than $0.5 \%$ and interactions were considered cross-validated if the difference in average percent variation predicted in independent test samples between the full model containing the interaction term and the reduced model containing only main effect terms was greater than $0.5 \%$.

To visualize the genetic architecture of ABI, we applied a novel data visualization scheme, the KGraph, described in Kelly et al. [32]. The KGraph was developed for the visualization of genetic association results and the underlying confounding due to SNP-SNP frequency correlations (i.e. LD), SNP-risk factor associations, and risk factor-risk factor correlations. It simultaneously displays both significant univariate associations and pairwise interactions 
with the outcome of interest, $\mathrm{ABI}$, as well as the underlying correlation structure among the predictor variables (SNPs and risk factors).

Using a SNP list that was comprised of SNPs that passed our three filters (FDR, replication, and cross-validation), multivariable linear regression models combining the top SNPs, risk factors, and their interactions were then constructed and the percent variation in ABI explained by each model was estimated. Four-fold cross validation was used to estimate the predictive ability of these models in test samples not used to estimate the models.

\section{Results}

The descriptive statistics for the full sample of non-Hispanic whites and the two subsets used to examine replication are presented in Table 1. The mean age was 59 years. The mean BMI was $31 \mathrm{~kg} / \mathrm{m}^{2}$. The average $\mathrm{ABI}$ was 1.11 . Fifteen percent of the participants had type II diabetes and $51 \%$ had a history of smoking.

In Table 2, we present a summary of the results from testing for SNP main effects, SNP-covariate, and SNP-SNP interactions and the number of associations that remained significant after adjustment for multiple testing (FDR $<0.3$ ), testing for replication, and cross-validation.
For example, 435 SNPs were evaluated for their association with adjusted $\mathrm{ABI}$ and 20 had FDR $<0.3,3$ internally replicated, and 5 cross-validated. Only two SNPs (located in the NOS3 gene, rs891512 and rs1808593) passed all three filters. In contrast, there were 6,926 tests of SNP-risk factor interactions and 20 had a FDR $<0.3,72$ internally replicated, but only 52 cross-validated. Only three SNPrisk factor interactions passed all three criteria - specifically, ADRB2_rs1042713 interacting with $\operatorname{Lp}(\mathrm{a})$ and SLC4A5 polymorphisms interacting with diabetes (Table 3 ). There were 91,113 tests of SNP-SNP interactions and we found 270 had a FDR $<0.3,973$ internally replicated, and 404 cross-validated. Only 25 SNP-SNP interactions passed all three criteria and are listed in Table 3.

Figure 1 is a visual representation of the complex genetic and demographic/biochemical risk factor associations underlying variation in ABI. Using both color and spatial relationships, the KGraph presents both associations with $\mathrm{ABI}$ and the correlation structure of the predictors that underlie those associations. Only SNPs that passed all three filters are displayed, though all SNP-ABI, SNP-SNP (i.e. LD), and SNP-risk factor associations are represented to more fully understand the complex correlation structure underlying $\mathrm{ABI}$ predictors. Region 1, shown in green, displays the association between the SNPs and biochemi-

Table I: Descriptive Statistics for Study Participants

\begin{tabular}{|c|c|c|c|c|}
\hline & Full Sample $(N=1046)$ & Subset I $(N=330)$ & Subset $2(N=329)$ & $\begin{array}{c}\text { Univariate association with } A B I \text { in ful } \\
\text { sample }(N=1046)\end{array}$ \\
\hline & Mean ( \pm SD) & Mean ( \pm SD) & Mean ( \pm SD) & $\beta$ estimate \\
\hline$A B I$ & $\mathrm{I} . \mathrm{I}(0.1)$ & I.I (0.2) & $\mathrm{I} . \mathrm{I}(0 . \mathrm{I})$ & \\
\hline Age, years & $59.1(10.1)$ & $60.8(9.1)$ & $61.3(9.0)$ & $-3.6 \mathrm{E}-03 * * * *$ \\
\hline $\mathrm{BMI}, \mathrm{kg} / \mathrm{m}^{2}$ & $30.9(6.2)$ & $31.1(6.1)$ & $31.9(6.3)$ & $2.9 \mathrm{E}-03 * * *$ \\
\hline Waist Hip Ratio, cm & $0.91(.11)$ & $0.92(0.1)$ & $0.92(0.1)$ & I.IEOI* \\
\hline $\mathrm{SBP}, \mathrm{mm} \mathrm{Hg}$ & $131.1(16.6)$ & $135.1(17.4)$ & $135.0(16.3)$ & $-1.6 \mathrm{E}-03 * * *$ \\
\hline $\mathrm{DBP}, \mathrm{mm} \mathrm{Hg}$ & $74.0(9.0)$ & $74.9(9.7)$ & $74.5(9.2)$ & $1.8 \mathrm{E}-03 * * * *$ \\
\hline Pulse pressure, $\mathrm{mm} \mathrm{Hg}$ & $57.2(15.3)$ & $60.2(15.8)$ & $60.5(16.1)$ & $-2.5 \mathrm{E}-03 * * *$ \\
\hline Total cholesterol, mg/dL & $196.7(34.9)$ & $193.0(32.8)$ & $195.2(33.4)$ & $-2.5 \mathrm{E}-05$ \\
\hline HDL cholesterol, mg/dL & $51.4(14.9)$ & $49.8(14.3)$ & $50.8(14.0)$ & $-8.1 \mathrm{E}-04 * *$ \\
\hline LDL cholesterol, mg/dL & $113.8(31.8)$ & $125.1(36.5)$ & $126.1(36.6)$ & $2.8 \mathrm{E}-04$ \\
\hline Triglycerides, mg/dL & $159.9(96.1)$ & $163.8(97.7)$ & $163.0(90.0)$ & $-7.4 \mathrm{E}-05$ \\
\hline C-reactive protein, $\mathrm{mg} / \mathrm{L}$ & $0.45(0.7)$ & $0.50(0.9)$ & $0.45(0.5)$ & $-7.2 \mathrm{E}-03$ \\
\hline Fibrinogen, $\mathrm{mg} / \mathrm{dL}$ & $318.3(77.0)$ & $321.0(73.6)$ & $324.7(78.3)$ & $-1.7 \mathrm{E}-04 * *$ \\
\hline Homocysteine, umol/L & $9.9(2.7)$ & $10.2(2.8)$ & $10.1(2.8)$ & $-4.9 \mathrm{E}-03 * *$ \\
\hline $\mathrm{Lp}(\mathrm{a}), \mathrm{mg} / \mathrm{dL}$ & $40.1(38.7)$ & $40.5(36.5)$ & $40.4(40.5)$ & $-4.6 \mathrm{E}-04 * *$ \\
\hline Female, n (\%) & $580(55 \%)$ & $170(52 \%)$ & $183(56 \%)$ & $-5.4 \mathrm{E}-02 * * *$ \\
\hline Ever smoker, n (\%) & $528(51 \%)$ & 174 (53\%) & 161 (49\%) & $-1.4 \mathrm{E}-02$ \\
\hline Diabetes, n (\%) & $154(15 \%)$ & $48(15 \%)$ & $71(22 \%)$ & $-2.4 \mathrm{E}-02$ \\
\hline Hypertensive, n (\%) & $773(74 \%)$ & $330(100 \%)$ & $329(100 \%)$ & $-3.7 \mathrm{E}-02 * * *$ \\
\hline
\end{tabular}

$*_{p}<0.05 ; * * p<0.01 ; * * * p<0.001$

$A B I$, ankle-brachial index; BMI, body mass index; SBP, systolic blood pressure; DBP, diastolic blood pressure; HDL, high density lipoprotein; LDL, low density lipoprotein; $L p(a)$, lipoprotein 
Table 2: Quantitative summary of genetic associations with ABI that replicated, cross-validated, and passed FDR criterion

\begin{tabular}{lccc}
\hline & SNP Main Effects & SNP-Covariate Interactions & SNP-SNP Interactions \\
\hline Number of tests & 435 & 6926 & 91113 \\
$P<0.10$ on full sample & 77 & 815 & 10308 \\
FDR $(<0.30)$ on full sample & 20 & 20 & 270 \\
Cross Validation (on full sample) & 5 & 52 & 404 \\
Replication (P<0.10 in both groups) & 3 & 72 & 973 \\
FDR and Cross-validation & 5 & 14 & 79 \\
FDR and Replication & 2 & 4 & 36 \\
Replication and Cross-validation & 2 & 7 & 52 \\
FDR and Cross-Validation and Replication & 2 & 3 & 25
\end{tabular}

FDR, False Discovery Rate

cal risk factors, one source of often overlooked confounding and information about underlying metabolic pathways. In this region, the cross-validated SNP associations with log triglyceride (TGFB3 and SLC22A3), log CRP
(ADD2), fibrinogen (ATP6B1), homocysteine (SLC17A2 and PKRAR2B), and $\operatorname{Lp}(\mathrm{a})$ (SLC22A3) are indicated. Region 2, shown in grey, illustrates the correlations between the risk factors. The majority of the risk factors

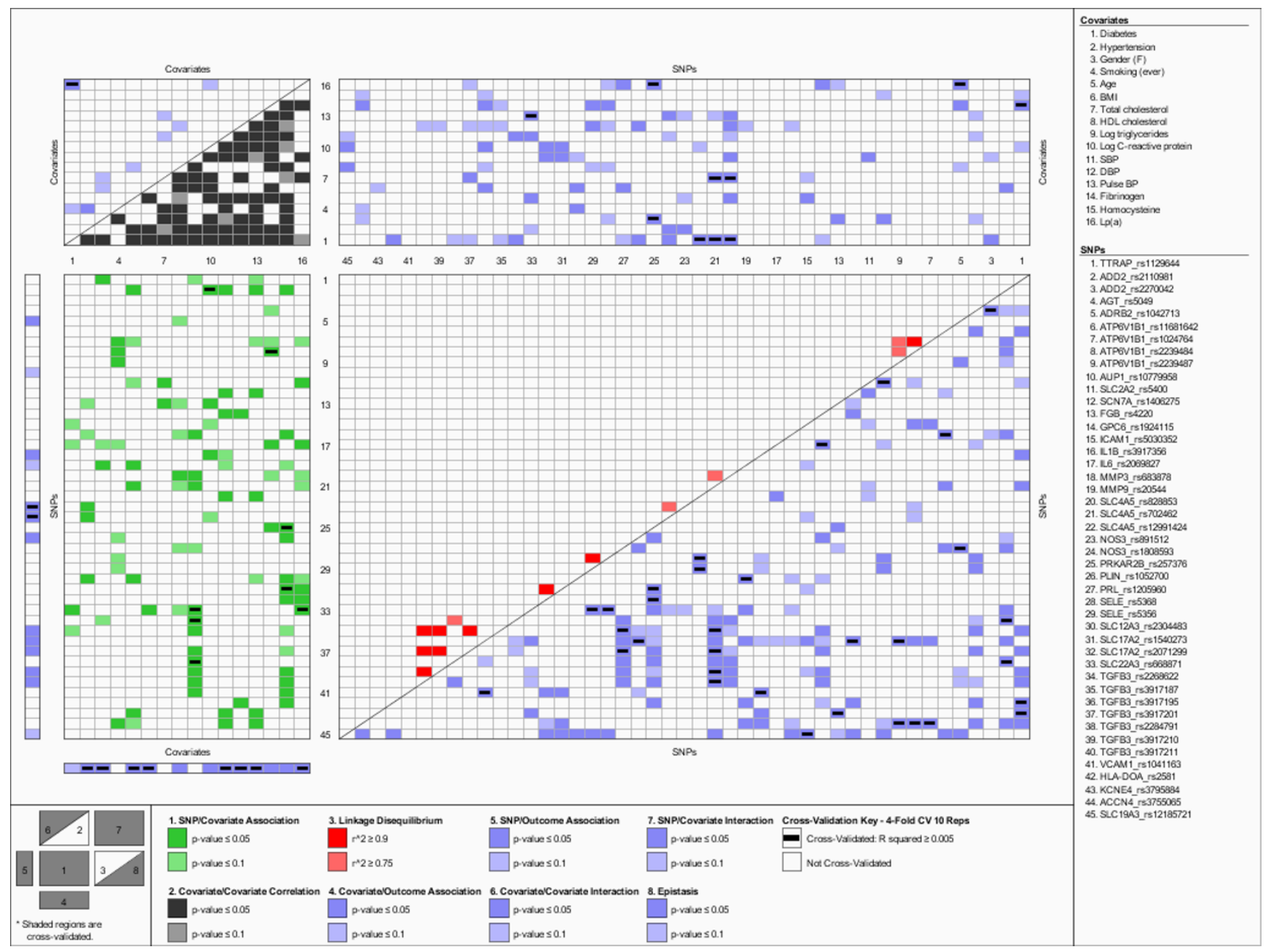

Figure I

Genetic architecture of the ankle-brachial index in non-Hispanic Whites. 
Table 3: Genetic effects that replicated, cross-validated, and passed FDR criterion

\begin{tabular}{|c|c|c|c|c|c|c|c|}
\hline Main Effects (2) & & SNP & Subset I p-value & Subset 2 p-value & $\begin{array}{l}\text { Full Sample p- } \\
\text { value }\end{array}$ & $\mathbf{R}^{2}$ & CV $R^{2}$ \\
\hline & & NOS3_rs8915I2 & 0.0003 & 0.0458 & 0.0005 & 0.0151 & 0.0096 \\
\hline & & NOS3_rsI808593 & 0.0006 & 0.0333 & 0.0001 & 0.0178 & 0.0105 \\
\hline \multirow{4}{*}{$\begin{array}{l}\text { SNP-Covariate } \\
\text { Interactions (3) }\end{array}$} & SNP & Covariate & & & & $\mathbf{R}^{2}$ & CV $R^{2}$ \\
\hline & ADRB2_rs I0427I3 & $\operatorname{Lp}(a)$ & 0.0847 & 0.0407 & 0.0006 & 0.0176 & 0.0089 \\
\hline & SLC4A5_rs828853 & DIABETES & 0.0564 & 0.0462 & 0.0004 & 0.0143 & 0.0095 \\
\hline & $\begin{array}{l}\text { SLC4A5_rs|299|42 } \\
4\end{array}$ & DIABETES & 0.0350 & 0.0278 & 0.0005 & 0.0106 & 0.0085 \\
\hline \multirow{26}{*}{$\begin{array}{l}\text { SNP-SNP } \\
\text { Interactions (25) }\end{array}$} & SNPI & SNP2 & & & & $\mathbf{R}^{2}$ & CV R $\mathbf{R}^{2}$ \\
\hline & TTRAP_rsII 29644 & HLA-DOA_rs258I & 0.0535 & 0.0608 & 0.0001 & 0.0198 & 0.0116 \\
\hline & ADD2_rs2II098I & TGFB3_rs2268622 & 0.0355 & 0.0134 & 0.0004 & 0.0171 & 0.0115 \\
\hline & ADD2_rs211098I & TGFB3_rs228479I & 0.0439 & 0.0049 & 0.0001 & 0.0200 & 0.0156 \\
\hline & $A D D 2 \_r s 2270042$ & $A G T \_r s 5049$ & 0.0085 & 0.0765 & 0.0003 & 0.0171 & 0.0127 \\
\hline & ADRB2_rs I0427I3 & PRL_rs|205960 & 0.0066 & 0.0885 & 0.0003 & 0.0175 & 0.0119 \\
\hline & $\begin{array}{l}\text { ATP6VIBI_rs|I68I } \\
642\end{array}$ & ILIB_rs39|7356 & 0.0223 & 0.0105 & 0.0002 & 0.0195 & 0.0130 \\
\hline & $\begin{array}{l}\text { ATP6VIBI_rsI0247 } \\
64\end{array}$ & ACCN4_rs3755065 & 0.0395 & 0.0005 & 0.0002 & 0.0189 & 0.0152 \\
\hline & $\begin{array}{l}\text { ATP6VIBI_rs22394 } \\
84\end{array}$ & ACCN4_rs3755065 & 0.0550 & 0.0005 & 0.0005 & 0.0161 & 0.0121 \\
\hline & $\begin{array}{l}\text { ATP6VIBI_rs22394 } \\
87\end{array}$ & ACCN4_rs3755065 & 0.0238 & 0.0013 & 0.0004 & 0.0175 & 0.0123 \\
\hline & AUPI_rsI0779958 & SLC2A2_rs5400 & 0.0041 & 0.0868 & 0.0003 & 0.0155 & 0.0149 \\
\hline & SCN7A_rsI406275 & TGFB3_rs3917195 & 0.0283 & 0.0004 & 0.0001 & 0.0180 & 0.0137 \\
\hline & FGB_rs 4220 & KCNE4_rs3795884 & $8.98 \mathrm{E}-05$ & 0.0584 & $3.45 \mathrm{E}-05$ & 0.0208 & 0.0163 \\
\hline & GPC6_rs1924II5 & IL6_rs2069827 & 0.0203 & 0.0338 & 0.0007 & 0.0145 & 0.0124 \\
\hline & ICAMI_rs5030352 & $\begin{array}{l}\text { SLCI9A3_rsI21857 } \\
21\end{array}$ & 0.0869 & 0.0028 & 0.0001 & 0.0172 & 0.0073 \\
\hline & MMP3_rs683878 & VCAMI_rsI04II63 & 0.0644 & 0.0407 & 0.0003 & 0.0172 & 0.0085 \\
\hline & MMP9_rs20544 & $\underset{3}{S L C I} 2 A \overline{3} \_r s 230448$ & 0.0157 & 0.0876 & 0.0002 & 0.0180 & 0.0132 \\
\hline & SLC4A5_rs702462 & TGFB3_rs3917187 & 0.0734 & 0.0010 & 0.0004 & 0.0176 & 0.0088 \\
\hline & SLC4A5_rs702462 & TGFB3_rs391720I & 0.0456 & 0.0010 & 7.65E-05 & 0.0207 & 0.0147 \\
\hline & SLC4A5_rs702462 & TGFB3_rs3917210 & 0.0329 & 0.0009 & 4.62E-05 & 0.0222 & 0.0156 \\
\hline & SLC4A5_rs702462 & TGFB3_rs391721I & 0.0358 & 0.0015 & $5.91 \mathrm{E}-05$ & 0.0212 & 0.0097 \\
\hline & PRKAR2B_rs257376 & $\begin{array}{l}\text { SLCI 7A2_rsI54027 } \\
3\end{array}$ & 0.0075 & 0.0036 & 0.0005 & 0.0174 & 0.0134 \\
\hline & PRKAR2B_rs 257376 & $\begin{array}{l}\text { SLCI 7A2_rs207II29 } \\
9\end{array}$ & 0.0161 & 0.0020 & 0.0002 & 0.0182 & 0.0103 \\
\hline & PLIN_rs 1052700 & TGFB3_rs3917195 & 0.0126 & 0.0015 & 0.0006 & 0.0146 & 0.0054 \\
\hline & SELE_rs5368 & SLC22A3_rs66887I & 0.0004 & 0.0954 & 0.0005 & 0.0167 & 0.0127 \\
\hline & SELE_rs5356 & SLC22A3_rs66887I & 0.0009 & 0.0932 & 0.0006 & 0.0157 & 0.0124 \\
\hline
\end{tabular}

All associations adjusted for age, gender, body mass index, smoking, diabetes, and hypertension.

ACCN4 amiloride-sensitive cation channel 4, pituitary; ADD2 adducin 2 (beta); ADRB2 *b2-adrenergic receptor; AGT angiotensinogen (serine (or cysteine) proteinase inhibitor, clade A (alpha-I antiproteinase, antitrypsin), member 8); ATP6VIBI ATPase, H+ transporting, lysosomal 56/58 kDa, VI subunit B, isoform I (Renal tubular acidosis with deafness); AUPI ancient ubiquitous protein I; FGB fibrinogen, $B$ beta polypeptide; GPC6 glypican 6; HLA-DOA human leukocyte antigen; ICAMI intercellular adhesion molecule I (CD54), human rhinovirus receptor; ILBI interleukin I, beta; IL6 interleukin 6 (interferon, beta 2); KCNE4 potassium voltage-gated channel, Isk-related family, member 4; MMP3 matrix metalloproteinase 3 (stromelysin I, progelatinase); MMP9 matrix metalloproteinase 9 (gelatinase B, $92 \mathrm{kDa}$ gelatinase, $92 \mathrm{kDa}$ type IV collagenase); NOS3 nitric oxide synthase 3 (endothelial cell); PLIN perilipin; PRKAR2B protein kinase, cAMP-dependent, regulatory, type II, beta; PRL prolactin; SCN7A sodium channel, voltage-gated, type VII, alpha; SELE selectin E (endothelial adhesion molecule I); SLC2A2 solute carrier family 2 (facilitated glucose transporter), member 2; SLC2A5 solute carrier family 4, sodium bicarbonate cotransporter, member 5; SLCI $2 A 3$ solute carrier family 12 (sodium/ chloride transporters), member 3; SLCI 7A2 solute carrier family 17 (sodium phosphate), member 2; SLCI 9A3 solute carrier family 19, member 3; SLC22A3 solute carrier family 22 (extraneuronal monoamine transporter), member 3; TGFB3 transforming growth factor, beta 3; TTRAP TRAF and TNF receptor associated protein; VCAMI vascular cell adhesion molecule I. 
are significantly correlated $(|\mathrm{r}|<0.3)$, with only Lp(a) levels not being highly correlated with other risk factors. The observed LD, shown in red in Region 3, occurs between SNPs that are within the same gene, with SNPs in the TGFB3, SELE, NOS3, and SLC4A5 genes being highly correlated.

The remaining regions are colored blue, indicating that they represent associations with ABI. Region 4, which displays the univariate association between risk factors and ABI shows that age, BMI, gender, hypertension, SBP, DBP, pulse pressure, and $\operatorname{Lp}(\mathrm{a})$ each have statistically significant and cross-validated associations with ABI. Region 5, which illustrates univariate associations between the SNPs and ABI, reveals that only two SNPs in NOS3 (which are in LD) have significant, replicated and cross-validated associations. Region 6 displays the risk factor-risk factor interactions significantly associated with ABI. Cross-validated interactions were observed between diabetes status and $\operatorname{Lp}(\mathrm{a})$. Region 7 displays the interactions between the SNPs and risk factors that were associated with ABI. Overall, we detected 10 statistically significant interactions between a variety of risk factors and SNPs that replicated and cross-validated. Upon controlling for multiple testing with FDR, only 3 risk factor-SNP interactions met our criteria. Namely, two SNPs within the SLC4A5 gene (rs828853 and rs12991424) interacted with diabetes, and one SNP within the ADRB2 gene (rs1042713) interacted with $\operatorname{Lp}(\mathrm{a})$. Region 8 displays the epistatic (SNP-SNP) interactions significantly associated with ABI. We detected 32 replicated and cross-validated, statistically significant pairwise interactions between SNPs. This number was reduced to 25 interactions after controlling for multiple testing with FDR. Approximately half of these interactions involved variants in the solute-carrier genes ( 7 interactions) and TGFB3 gene ( 5 interactions).

To begin to assess the combined predictive ability of the top SNPs, risk factors, and their interactions, we used multivariable modeling techniques and investigated the percent variation in ABI explained in the full sample and in the independent test samples used in the cross validation (i.e. a more accurate estimate of the predictive ability of these variations for other yet to be sampled individuals in this population of inference) (see Table 4). We found that the two single SNPs that met our criteria explained 0.65 percent of variation (adjusted $\mathrm{R}^{2}$ ) in $\mathrm{ABI}$ alone (not adjusting for risk factors) and the top four SNP-SNP interactions explained an additional $4.5 \%$ of variation in $\mathrm{ABI}$. The covariates explained $12.5 \%$ of the variability in $\mathrm{ABI}$ alone while the top SNP-covariate interactions explained an additional $2.25 \%$ (adjusted $\mathrm{R}^{2}=15.04$ ). After accounting for risk factors and their interactions with SNPs, the top SNP-SNP interactions explained an additional 1.75\%. Combining the top SNPs, risk factors, and their interactions into a model explained $17.85 \%$ of variation in ABI in the sample. To assess the predictive ability of these models in new individuals from the same population we used cross-validation methods and estimated the CV R2 (see Methods). The predictive ability of the genetic variations appears to be modest, at best, compared to the covariates.

\section{Discussion}

Multiple studies have investigated the association of polymorphisms in candidate genes with essential hypertension and coronary heart disease, but relatively few studies have explored the relationship between specific candidate gene polymorphisms and ABI, a marker of PAD. Our motivating hypothesis was that genetic polymorphisms implicated in risk factors for hypertension and CHD may influence PAD risk by means of common pathophysiological pathways. Therefore, in order to understand the genetic architecture of a complex multifactorial trait such as ABI, larger scale investigations of the polygenic network of genes and their impact on underlying physiological and biochemical correlates need to be examined simultaneously [33]. Out of 112 biological and positional candidate genes, SNPs in 30 different genes were related to inter-individual variation in $\mathrm{ABI}$, a non-invasive measure of PAD, in our study. Six of these genes were also associated with underlying physiological correlates.

Even after adjustment for conventional risk factors and stringent type I error reduction techniques, two of the NOS3 SNPs shared significant associations with ABI, sug-

Table 4: Multivariable analysis to assess combined predictive ability of the best SNPs, risk factors, and interactions

\begin{tabular}{|c|c|c|}
\hline Model & Adj $\mathbf{R}^{2}$ & CV $\mathbf{R}^{2}$ \\
\hline Single Replicated and CVD SNPs & 0.0065 & 0.0009 \\
\hline Single SNPs + Top 4 SNP*SNP Interactions & 0.0515 & 0.0066 \\
\hline CVD Covariates & 0.1252 & 0.1040 \\
\hline CVD Covariates + Single SNPs & 0.1397 & 0.1178 \\
\hline CVD Covariates + Single SNPs + 3 SNP*Covariate Interactions & 0.1504 & 0.1118 \\
\hline CVD Covariates + Single SNPs + Top 4 SNP*SNP Interactions & 0.1652 & 0.0877 \\
\hline CVD Covariates + Single SNPs + 3 SNP*Covariate Interactions + Top 4 SNP*SNP Interactions & 0.1785 & 0.0963 \\
\hline
\end{tabular}

CVD, cross-validated; SNPs, Single Nucleotide Polymorphisms 
gesting that alterations in NOS3 may indeed influence inter-individual variation in $\mathrm{ABI}$. We did genotype the well-known NOS3 non-synonymous SNP Asp298Glu (rs1799983), which has been postulated to alter function of NOS3 [34], but did not find the SNP to be associated with ABI. These findings are consistent with our previous report of an association between polymorphisms in NOS3 and inter-individual variation in ABI [7].

Diabetes is one of the main risk factors for PAD. Several studies have identified genetic variants that increase risk for PAD among type 2 diabetics [35-37]. As such, it is plausible that genetic susceptibility to PAD is modified by diabetes status. In line with this, 2 of the 3 SNP-covariate interactions that passed our stringent criteria involved diabetes as the environmental covariate. While the prevalence of diabetes was low in our sample, our results provide preliminary evidence for a gene-environment interaction, even after adjustment for conventional risk factors. This finding underscores the importance of considering the particular contexts that may potentially modify genetic susceptibility to complex disease.

An interesting finding from our study is that the majority of significant genetic effects were in the form of epistatic interactions. This finding provides further evidence that the genetic susceptibility to complex atherosclerotic diseases is not attributable to the modest effects of a single gene and is likely a result of a combination of alleles in multiple genes [6]. Animal and plant studies have also recently shown an abundance of epistatic interactions, more than had previously been expected [38].

In the clinical setting, $\mathrm{ABI}$ is used as a dichotomous variable, with cut off values of $\leq 0.90$ or $\leq 0.95$ employed to confirm the presence of PAD. We did not analyze ABI as a dichotomous variable as this entailed a substantial loss of statistical power, particularly since the prevalence of an abnormal ABI (defined as $\leq 0.90$ ) was low $(6.8 \%$ ) in our study sample. Despite this, our analyses with ABI as a continuous outcome were warranted as recent studies suggest that, even in the range of 1.0-1.3, lower ABI may be related to PAD risk factors [39]. Furthermore, just as genetic variation influencing $\mathrm{BP}$ variation in normotensives has been related to an increased risk of hypertension [40], we expected that genetic variation associated with $\mathrm{ABI}$ levels might be related to an increased risk of PAD.

An interesting result from this study is the relatively low level of agreement between results filtered through different methods of reducing false positives - namely, adjustment for multiple testing using FDR $<0.30$, internal replication, and four-fold cross-validation. One of the shortcomings of genetic association studies is that they have often failed to replicate and Manly [10] suggests that internal validation, common to good experimental practices, is one way to avoid the publication of false positives. In our study, we used cross-validation methods to significantly reduce the chance of false positives. Cross-validation methods were developed in the late 1970's as a way to incorporate a measure of predictive accuracy (and correspondingly, a measure of prediction error) for an estimated model based on its performance predicting the outcome for independent test cases [12]. During the last decade, cross-validation methods have been used widely for everything from robust variable selection in gene expression array studies [41] to reducing false positives in gene-gene interaction studies $[42,43]$ to evaluating the predictive accuracy of molecular or genetic classifiers of disease before clinical implementation [44]. Cross-validation has become a standard in the field of metabolomic [45], proteomic [46,47], and transcriptomic [48] studies because of its ease of execution and its emphasis on prediction in independent test cases as a method of discriminating between true associations and false associations.

We should note that although it appeared in this study that FDR was more conservative than cross-validation or internal replication, this is not always the case. We have conducted similar analyses in other studies (results not shown) and have found cross-validation to be more conservative than the FDR, leading us to the general conclusion that multiple methods should be employed simultaneously to reduce type I errors for genetic association studies.

Concerns have been raised that population stratification may lead to spurious results in genetic association studies [44]. To address this potential impact, we assessed the presence of population substructure using STRUCTURE [49] and found no evidence of subpopulation clusters in our sample. Wacholder et al. have pointed out that "population stratification does not occur in an ethnically homogeneous population" [50] and the bias that may arise in a population-based study of non-Hispanic Caucasians, as a result of ignoring ethnicity, is likely to be very small [51].

Some limitations of the present study need to be considered. Our approach was based on the premise that susceptibility alleles for common diseases (and related subclinical disease measures such as $\mathrm{ABI}$ ) are not under strong negative selection, and common variants contribute to common disease traits (i.e. the 'common disease common variant' hypothesis) [52]. However, the allelic spectrum for genes associated with complex quantitative traits such as ABI is not fully delineated, and it is possible that multiple rare polymorphisms in the biological and positional candidate genes that we studied influence ABI. Due to a lack of power, identifying association with $\mathrm{ABI}$ 
using such alleles would not be possible using the approaches employed in this study. Our inferences may not be generalizable to individuals who are younger, normotensive, or of other ethnicities. Although a priori power calculations indicated that we were adequately powered to detect relatively small SNP effects, insufficient sample sizes (full sample and re-sampled subsets) or random measurement error may have limited our power to detect genotype-phenotype associations. Despite some limitations, our approach illustrates the use of SNPs in candidate genes to construct a more complete picture of the genetic architecture of complex traits such as ABI.

\section{Conclusion}

The genetic architecture of complex multifactorial traits includes common genetic variants with small effects as well as gene-gene and gene-environment interactions. We report that candidate gene SNP main effects, SNP-covariate and SNP-SNP interactions contribute to the inter-individual variation in $\mathrm{ABI}$, a marker of PAD. Our findings underscore the importance of conducting systematic investigations that consider a context-dependent framework for developing a deeper understanding of the multidimensional genetic and environmental factors that contribute to complex diseases.

\section{Competing interests}

The authors declare that they have no competing interests.

\section{Authors' contributions}

SLRK and IJK had the original idea of this article, performed the design, participated in the discussion of the results, and wrote the manuscript. MTG performed the analyses, performed the design, participated in the discussion of the results, and wrote the manuscript. EB and STT participated in the discussion of the results.

\section{Additional material}

\section{Additional file 1}

Summary of Genotyped SNPs in Candidate Genes.

Click here for file

[http://www.biomedcentral.com/content/supplementary/17558794-1-16-S1.pdf]

\section{Acknowledgements}

This work was supported by grant HL75794, HL68737, HL05448I, HL54457, and the General Clinical Research Center Grant M0I RR00585 from National Institutes of Health.

\section{References}

I. Hiatt WR, Hoag S, Hamman RF: Effect of diagnostic criteria on the prevalence of peripheral arterial disease. The San Luis Valley Diabetes Study. Circulation 1995, 91 : 1472-1479.
2. Criqui $\mathrm{MH}$ : Peripheral arterial disease - epidemiological aspects. Vasc Med 200I, 6:3-7.

3. McDermott MM, Fried L, Simonsick E, Ling S, Guralnik JM: Asymptomatic peripheral arterial disease is independently associated with impaired lower extremity functioning: The women's health and aging study. Circulation 2000, I0I:I007-I0I2.

4. Cotran RS, Kumar V, Collins T, (Eds): Pathologic Basis of Disease Philadelphia: WB Saunders Co.; 1999.

5. Kullo IJ, Bailey KR, Kardia SL, Mosley TH Jr, Boerwinkle E, Turner ST: Ethnic differences in peripheral arterial disease in the NHLBI Genetic Epidemiology Network of Arteriopathy (GENOA) study. Vasc Med 2003, 8:237-242.

6. Knowles JW, Assimes TL, Li J, Quertermous T, Cooke JP: Genetic susceptibility to peripheral arterial disease: a dark corner in vascular biology. Arterioscler Thromb Vasc Biol 2007, 27:2068-2078.

7. Kullo IJ, Greene MT, Boerwinkle E, Chu J, Turner ST, Kardia SL: Association of polymorphisms in NOS3 with the ankle-brachial index in hypertensive adults. Atherosclerosis 2007.

8. Hirschhorn JN, Altshuler D: Once and again-issues surrounding replication in genetic association studies. J Clin Endocrinol Metab 2002, 87:4438-444I.

9. Sing CF, Stengard JH, Kardia SL: Genes, environment, and cardiovascular disease. Arterioscler Thromb Vasc Biol 2003, 23: I190-II96.

10. Manly KF: Reliability of statistical associations between genes and disease. Immunogenetics 2005, 57:549-558.

1I. Storey JD: A direct approach to false discovery rates. Journal of the Royal Statistical Society 2002, Series B, 64:479-498.

12. Stone M: Cross-validatory choice and assessment of statistical predictions. Journal of the Royal Statistical Society 1974, Series B, 36:1II-147.

13. FBPP Investigators: Multi-center genetic study of hypertension: The Family Blood Pressure Program (FBPP). Hypertension 2002, 39:3-9.

14. Daniels PR, Kardia SL, Hanis CL, Brown CA, Hutchinson R, Boerwinkle E, Turner ST, Genetic Epidemiology Network of Arteriopathy study: Familial aggregation of hypertension treatment and control in the Genetic Epidemiology Network of Arteriopathy (GENOA) study. Am J Med 2004, I | 6:676-68I.

15. Friedewald WT, Levy RI, Fredrickson DS: Estimation of the concentration of low-density lipoprotein cholesterol in plasma, without use of the preparative ultracentrifuge. Clin Chem 1972, 18:499-502.

16. Keevil BG, Nicholls SP, Kilpatrick ES: Evaluation of a latexenhanced immunoturbidimetric assay for measuring low concentrations of C-reactive protein. Ann Clin Biochem 1998, 35(Pt 5):67|-673.

17. von Clauss A: Gerinnungsphysiologische schnellmethode zur bestimmung des fibrinogens. Acta Haematol 1957, 17:237-246.

18. Kullo IJ, Bailey KR, Bielak LF, Sheedy PF 2nd, Klee GG, Kardia SL, Peyser PA, Boerwinkle E, Turner ST: Lack of association between lipoprotein(a) and coronary artery calcification in the Genetic Epidemiology Network of Arteriopathy (GENOA) study. Mayo Clin Proc 2004, 79:1258-1263.

19. Barkley RA, Chakravarti A, Cooper RS, Ellison RC, Hunt SC, Province MA, Turner ST, Weder AB, Boerwinkle E, Family Blood Pressure Program: Positional identification of hypertension susceptibility genes on chromosome 2. Hypertension 2004, 43:477-482.

20. dbSNP [http://www.ncbi.nlm.nih.gov/SNP/]

21. Seattle SNPs [http://pga.mbt.washington.edu]

22. Carlson CS, Eberle MA, Rieder MJ, Yi Q, Kruglyak L, Nickerson DA: Selecting a maximally informative set of single-nucleotide polymorphisms for association analyses using linkage disequilibrium. Am J Hum Genet 2004, 74: 106-I 20.

23. $R$ Core Development Team: $A$ language and environment for statistical computing. 2005.

24. Lynch M, Walsh B: Genetics and Analysis of Quantitative Traits Sudnerland, MA: Sinauer Associates, Inc.; 1998.

25. Weir B: Genetic Data Analysis II Massachusetts: Sinauer Associates; 1996.

26. Shammas NW: Epidemiology, classification, and modifiable risk factors of peripheral arterial disease. Vasc Health Risk Manag 2007, 3:229-234.

27. Hirsch AT, Criqui MH, Treat-Jacobson D, Regensteiner JG, Creager MA, Olin JW, Krook SH, Hunninghake DB, Comerota AJ, Walsh ME, 
McDermott MM, Hiatt WR: Peripheral arterial disease detection, awareness, and treatment in primary care. JAMA 200I, 286: $1317-1324$.

28. Meijer WT, Hoes AW, Rutgers D, Bots ML, Hofman A, Grobbee DE: Peripheral arterial disease in the elderly: The Rotterdam Study. Arterioscler Thromb Vasc Biol 1998, 18:185-192.

29. Newman AB, Siscovick DS, Manolio TA, Polak J, Fried LP, Borhani NO, Wolfson SK: Ankle-arm index as a marker of atherosclerosis in the Cardiovascular Health Study. Cardiovascular Heart Study (CHS) Collaborative Research Group. Circulation 1993, 88:837-845.

30. Kleinbaum D, Kupper L, Muller K, Nizam A: Applied Regression Analysis and Other Multivariate Methods Pacific Grove, CA: Brooks/Cole Publishing Company; 1998.

31. Molinaro AM, Simon R, Pfeiffer RM: Prediction error estimation: A comparison of resampling methods. Bioinformatics 2005, 2I:330I-3307.

32. Kelly RJ, Jacobsen DM, Sun YV, Smith JA, Kardia SL: A system for visualizing and evaluating complex genetic associations. Bioinformatics 2007, 23:249-25I.

33. Churchill GA: Recombinant inbred strain panels: A tool for systems genetics. Physiol Genomics 2007.

34. Wattanapitayakul SK, Mihm MJ, Young AP, Bauer JA: Therapeutic implications of human endothelial nitric oxide synthase gene polymorphism. Trends Pharmacol Sci 200I, 22:36I-368.

35. Resnick HE, Rodriguez B, Havlik R, Ferrucci L, Foley D, Curb JD, Harris TB: Apo E genotype, diabetes, and peripheral arterial disease in older men: The Honolulu Asia-aging study. Genet Epidemiol 2000, 19:52-63.

36. Pollex RL, Mamakeesick M, Zinman B, Harris SB, Hanley AJ, Hegele RA: Methylenetetrahydrofolate reductase polymorphism $677 \mathrm{C}>\mathrm{T}$ is associated with peripheral arterial disease in type 2 diabetes. Cardiovasc Diabetol 2005, 4: 17.

37. Libra M, Signorelli SS, Bevelacqua Y, Navolanic PM, Bevelacqua V, Polesel J, Talamini R, Stivala F, Mazzarino MC, Malaponte G: Analysis of G(-174)C IL-6 polymorphism and plasma concentrations of inflammatory markers in patients with type 2 diabetes and peripheral arterial disease. J Clin Pathol 2006, 59:2II-2I5.

38. Chevrud JM: Chapter 4. In Epistasis and the Evolutionary Process New York: Oxford University Press; 2000:58-59-8I.

39. McDermott MM, Liu K, Criqui MH, Ruth K, Goff D, Saad MF, Wu C, Homma S, Sharrett AR: Ankle-brachial index and subclinical cardiac and carotid disease: The multi-ethnic study of atherosclerosis. Am J Epidemiol 2005, 162:33-4I.

40. Chang YP, Liu K, Kim JD, Ikeda MA, Layton MR, Weder AB, Cooper RS, Kardia SL, Rao DC, Hunt SC, Luke A, Boerwinkle E, Chakravarti A: Multiple genes for essential hypertension susceptibility on chromosome Iq. Am J Hum Genet 2007, 80(2):253-264.

4I. Zhu J, Hastie T: Classification of gene microarrays by penalized logistic regression. Biostatistics 2004, 5:427-443.

42. Ritchie MD, Motsinger AA: Multifactor dimensionality reduction for detecting gene-gene and gene-environment interactions in pharmacogenomics studies. Pharmacogenomics 2005, 6:823-834

43. Gong R, Liu Z, Li L: Epistatic effect of plasminogen activator inhibitor I and beta-fibrinogen genes on risk of glomerular microthrombosis in lupus nephritis: Interaction with environmental/clinical factors. Arthritis Rheum 2007, 56:1608-1617.

44. Lander ES, Schork NJ: Genetic dissection of complex traits. Science 1994, 265:2037-2048.

45. Pohjanen E, Thysell E, Jonsson P, Eklund C, Silfver A, Carlsson IB, Lundgren $\mathrm{K}$, Moritz T, Svensson MB, Antti $\mathrm{H}$ : A multivariate screening strategy for investigating metabolic effects of strenuous physical exercise in human serum. J Proteome Res 2007, 6:2।|3-2। 20 .

46. Agranoff $D$, Fernandez-Reyes $D$, Papadopoulos MC, Rojas SA, Herbster M, Loosemore A, Tarelli E, Sheldon J, Schwenk A, Pollok R, Rayner CF, Krishna S: Identification of diagnostic markers for tuberculosis by proteomic fingerprinting of serum. Lancet 2006, 368: I012-102I.

47. Wood IA, Visscher PM, Mengersen KL: Classification based upon gene expression data: Bias and precision of error rates. Bioinformatics 2007, 23: 1363-1370.

48. Mertens BJ, De Noo ME, Tollenaar RA, Deelder AM: Mass spectrometry proteomic diagnosis: Enacting the double crossvalidatory paradigm. J Comput Biol 2006, I3:159|-1605.
49. Pritchard JK, Stephens M, Donnelly P: Inference of population structure using multilocus genotype data. Genetics 2000, 155:945-959.

50. Wacholder S, Rothman N, Caporaso N: Counterpoint: Bias from population stratification is not a major threat to the validity of conclusions from epidemiological studies of common polymorphisms and cancer. Cancer Epidemiol Biomarkers Prev 2002, I I:513-520.

51. Wacholder S, Rothman N, Caporaso N: Population stratification in epidemiologic studies of common genetic variants and cancer: Quantification of bias. J Natl Cancer Inst 2000, 92:II5I-II58.

52. Reich DE, Lander ES: On the allelic spectrum of human disease. Trends Genet 2001, 17:502-510.

\section{Pre-publication history}

The pre-publication history for this paper can be accessed here:

http://www.biomedcentral.com/1755-8794/1/16/prepub

Publish with Biomed Central and every scientist can read your work free of charge

"BioMed Central will be the most significant development for disseminating the results of biomedical research in our lifetime. "

Sir Paul Nurse, Cancer Research UK

Your research papers will be:

- available free of charge to the entire biomedical community

- peer reviewed and published immediately upon acceptance

- cited in PubMed and archived on PubMed Central

- yours - you keep the copyright

Submit your manuscript here:

http://www.biomedcentral.com/info/publishing_adv.asp
BioMedcentral 